\title{
Joule Heating Effect on MHD Combined Convection in a Wavy Chamber Having Conducting Square Cylinder
}

\author{
R. Nasrin* \\ Department of Mathematics, Bangladesh University of Engineering and Technology, \\ Dhaka-1000, Bangladesh
}

Received 14 July 2011, accepted in final revised form 1 December 2011

\begin{abstract}
The current simulation is focused on MHD combined convection flow and heat transfer characteristics in a square lid driven chamber. At the centre of this chamber a heat conducting solid square cylinder is located. Galerkin weighted residual finite element method is used to solve the governing equations of mass, momentum and energy. The left vertical wall of the chamber is mechanically lid driven and having temperature $T_{\text {cold }}$ and velocity $V_{0}$. But the right wall is sinusoidal wavy pattern and contains more temperature $\left(T_{\text {hot }}\right)$ than the left lid. The top and bottom surfaces are adiabatic. The behavior of the fluid for the values of Joule heating parameter $J(0,1,4,7)$ and Richardson number $R i(0.1,1$, $10)$ is described in details. The variations of the average Nusselt number $(\mathrm{Nu})$, the mean temperature of the fluid $\left(\theta_{a v}\right)$ and the temperature at cylinder centre $\left(\theta_{c}\right)$ for various $R i$ and $J$ are also presented. Maximum rate of heat transfer is occurred for the lowest $J$ at each Ri.
\end{abstract}

Keywords: MHD; Wavy chamber; Conducting cylinder; Combined convection; Finite element formulation.

(c) 2012 JSR Publications. ISSN: 2070-0237 (Print); 2070-0245 (Online). All rights reserved.

doi:10.3329/jsr.v4i1.8014 J. Sci. Res. 4 (1), 39-49 (2012)

\section{Introduction}

The influence of the magnetic field on the convective heat transfer and the mixed convection flow of the fluid are of paramount importance in engineering. When current flows in a wire, the resistance of the wire causes a voltage drop along the wire. As a result electrical energy is lost. This lost electrical energy is converted into thermal energy called Joule heating. Combined convection in lid driven chambers has received a sustained attention, due to the interest of the phenomenon in many technological processes, such as the design of solar collectors, nuclear reactor, lakes and reservoirs, crystal growth, thermal design of buildings, air conditioning and recently, the cooling of electronic circuit boards.

\footnotetext{
* Corresponding author: rehena@math.buet.ac.bd
} 
Analysis of a mixed convection flow usually requires an understanding of the two limiting regimes. The heat transfer from a wavy surface is functional in several practical appliances for instance refrigeration system, radiator, louver, plurality of holes etc. A combined free and forced convection flow of an electrically conducting fluid in a cavity in presence of magnetic and Joule heating effect is of special technical significance. This type of phenomena also takes place in electronic packages, micro electronic devices during their operations.

Combined free and forced convection in a square enclosure with heat conducting body and a finite-size heat source was simulated numerically by Hsu and How [1]. At the same time, Omri and Nasrallah [2] studied mixed convection in an air-cooled cavity with differentially heated vertical isothermal sidewalls having inlet and exit ports by a control volume finite element method. They investigated two different placement configurations of the inlet and exit ports on the sidewalls. Best configuration was selected analyzing the cooling effectiveness of the cavity, which suggested that injecting air through the cold wall was more effective in heat removal and placing inlet near the bottom and exit near the top produce effective cooling. The problem of unsteady laminar combined forced and free convection flow and heat transfer of an electrically conducting and heat generating or absorbing fluid in a vertical lid-driven cavity in the presence of a magnetic field was formulated by Chamkha [3]. They concluded that both the heat transfer coefficient and the dimensionless temperature in the body center strongly depended on the configurations of the system. Furthermore, Wang and Chen [4] analyzed forced convection in a wavy-wall channel and demonstrated the effects of wavy geometry, Reynolds number and Prandtl number on the skin friction and Nusselt number. Results were shown that the amplitudes of skin friction coefficient and Nusselt number had increased with an increase in the amplitude to wavelength ratio. Gau et al. [5] conducted mixed convection in rectangular cavities at various aspect ratios with moving isothermal side walls and constant flux heat source on the bottom wall.

Shokouhmand and Sayehvand [6] carried out the numerical study of flow and heat transfer in a square driven cavity. They found that at the higher values of Reynolds number, an inviscid core region developed, but secondary eddies were present in the bottom corners of the square at all Reynolds numbers. Bhoite et al. [7] studied numerically the problem of mixed convection flow and heat transfer in a shallow enclosure with a series of block-like heat generating component for a range of Reynolds and Grashof numbers and block-to-fluid thermal conductivity ratios. They showed that higher Reynolds number created a recirculation region of increasing strength at the core region and the effect of buoyancy became insignificant beyond a Reynolds number of typically 600, and the thermal conductivity ratio had a negligible effect on the velocity fields. Recently Al-Amiri et al. [8] investigated mixed convection heat transfer in liddriven cavity with a sinusoidal wavy bottom surface. Their findings were that the corrugated lid-driven cavity could be considered as an effective heat transfer mechanism at larger wavy surface amplitudes and low Richardson number. Very recently Nasrin [9] conducted combined magnetoconvection in a wavy enclosure with the effect of heat 
conducting cylinder, where finite element method was used to simulate the governing equations. At the same year, Nasrin [10] performed aspect ratio effect of vertical lid driven chamber having a centered conducting solid on mixed magnetoconvection. The author showed that maximum rate of heat transfer is observed for the lowest aspect ratio $A R$ owing to the shortest distance between the hot and cold surfaces.

In the light of the above literature review, it appears that a little significant work was reported on MHD combined convection in a lid driven cavity with a wavy heated surface in presence of heat conducting cylinder. The present study incorporates this issue with the effects of Joule heating parameter and Richardson number on the thermal and flow fields.

\section{Model Specification}

The geometry of the problem herein studied is depicted in Fig. 1. The system consists of a lid driven square chamber with sides of length $L$, within which a heat conducting cylinder is centered. The cavity is saturated with electrically conducting fluid of thermal conductivity $k$ where the cylinder has a thermal conductivity of $k_{s}$. The left vertical wall of the cavity is mechanically lid driven and considered to be at a constant temperature $T_{\text {cold }}$ and velocity $\mathrm{V}_{0}$. The wavy right vertical wall contains temperature $T_{\text {hot }}$. The corrugated wall is assumed more heated than the left lid. The top and bottom surfaces of the chamber are insulated. A transverse magnetic field of strength $B_{0}$ is imposed in the normal direction of the side walls. In addition Joule heating effect is considered here.

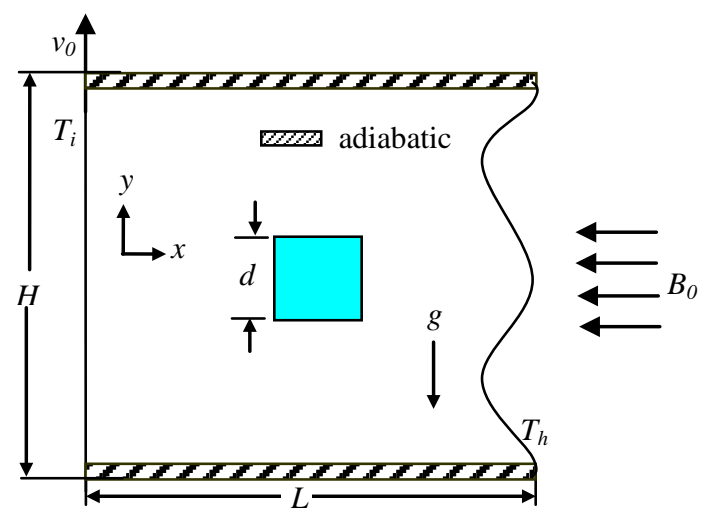

Fig. 1: Schematic diagram of the enclosure

\section{Mathematical Formulation}

The functioning fluid is assumed to be Newtonian, steady and incompressible with the flow is set to operate in the laminar mixed convection regime. The dimensionless equations describing the flow under Boussinesq approximation according to [11] are as follows: 


$$
\begin{aligned}
& \frac{\partial U}{\partial X}+\frac{\partial V}{\partial Y}=0 \\
& U \frac{\partial U}{\partial X}+V \frac{\partial U}{\partial Y}=-\frac{\partial P}{\partial X}+\frac{1}{R e}\left(\frac{\partial^{2} U}{\partial X^{2}}+\frac{\partial^{2} U}{\partial Y^{2}}\right) \\
& U \frac{\partial V}{\partial X}+V \frac{\partial V}{\partial Y}=-\frac{\partial P}{\partial Y}+\frac{1}{R e}\left(\frac{\partial^{2} V}{\partial X^{2}}+\frac{\partial^{2} V}{\partial Y^{2}}\right)+R i \theta-\frac{H a^{2}}{R e} V \\
& U \frac{\partial \theta}{\partial X}+V \frac{\partial \theta}{\partial Y}=\frac{1}{\operatorname{RePr}}\left(\frac{\partial^{2} \theta}{\partial X^{2}}+\frac{\partial^{2} \theta}{\partial Y^{2}}\right)+J V^{2} \\
& \left(\frac{\partial^{2} \theta_{s}}{\partial X^{2}}+\frac{\partial^{2} \theta_{s}}{\partial Y^{2}}\right)=0
\end{aligned}
$$

Here $\mathrm{Ha}$ is Hartmann number which is defined as $H a^{2}=\frac{\sigma B_{0}^{2} L^{2}}{\mu}$. Also $R e=\frac{V_{0} L}{v}$, $\operatorname{Pr}=\frac{v}{\alpha}, \quad R i=\frac{g \beta \Delta T L}{V_{0}^{2}}$ and $\quad J=\frac{\sigma B_{0}^{2} \alpha}{\rho C_{p}\left(T_{\text {hot }}-T_{\text {cold }}\right)}$ are Reynolds number, Prandtl number,

Richardson number and Joule heating parameter, respectively. Thermal diffusivity, volumetric thermal expansion coefficient, dynamic viscosity, kinematic viscosity, electrical conductivity, density and dimensional temperature difference of the fluid are represented by the symbols $\alpha, \beta, \mu, v, \sigma, \rho, \Delta T$, respectively.

By using the following dimensionless quantities, the Eqs. (1) - (5) are nondimensionalized:

$$
\begin{aligned}
& X=\frac{x}{L}, \quad Y=\frac{y}{L}, \quad U=\frac{u}{V_{0}}, \quad V=\frac{v}{V_{0}}, D=\frac{d}{L}, \\
& P=\frac{p}{\rho V_{0}^{2}}, \theta=\frac{\left(T-T_{\text {cold }}\right)}{\left(T_{\text {hot }}-T_{\text {cold }}\right)}, \theta_{S}=\frac{\left(T_{s}-T_{\text {cold }}\right)}{\left(T_{\text {hot }}-T_{\text {cold }}\right)}
\end{aligned}
$$

For the present problem, the boundary conditions are specified as follows:

at the sliding lid: $U=0, V=1, \theta=0$

at the vertical wavy surface: $U=0, V=0, \theta=1$

at the solid horizontal walls: $U=0, V=0, \frac{\partial \theta}{\partial N}=0$

at the square cylinder boundary: $U=0, V=0$

at the fluid-solid interface: $\left(\frac{\partial \theta}{\partial N}\right)_{\text {fluid }}=K\left(\frac{\partial \theta_{S}}{\partial N}\right)_{\text {solid }}$

Here $N$ is the non-dimensional distances either $X$ or $Y$ direction acting normal to the surface and $K$ is the dimensionless ratio of the thermal conductivity $\left(k_{s} / k\right)$. 
The nature of the vertical wavy surface profile is assumed the pattern of $X-1=-A[1-\cos (2 \lambda \pi Y)]$, where $A$ is the dimensionless amplitude of the wavy surface and $\lambda$ is the number of undulations.

The rate of heat transfer is computed at the wavy wall and is expressed in terms of the local Nusselt number $\overline{N u}$ as $\overline{N u}=\frac{h L}{k}=-\frac{\partial \theta}{\partial N} L$. The dimensionless normal temperature gradient can be written as $\frac{\partial \theta}{\partial N}=\frac{1}{L} \sqrt{\left(\frac{\partial \theta}{\partial X}\right)^{2}+\left(\frac{\partial \theta}{\partial Y}\right)^{2}}$.

The average Nusselt number $(\mathrm{Nu})$ is obtained by integrating the local Nusselt number along the vertical wavy surface and is defined by $N u=\frac{1}{S} \int_{0}^{S} \overline{N u} d N$, where $S$ is the total chord length of the wavy surface. The average temperature of the fluid is defined as $\theta_{a v}=\int \theta d \bar{V} / \bar{V}$, where $\bar{V}$ is the cavity volume.

\section{Computational Procedure}

The momentum and energy balance equations are the combination of mixed ellipticparabolic system of partial differential equations that have been solved by using the Galerkin weighted residual finite element technique of Rahman et al. [11]. The basic unknowns for the above differential equations are the velocity components $U, V$, the temperature $\theta$ and the pressure $P$. The velocity components, the temperature profile and linear interpolation for the pressure distribution according to their highest derivative orders in the differential Eqs. (2) - (5) are as

$$
\begin{aligned}
& U(X, Y)=N_{\beta} U_{\beta}, \quad V(X, Y)=N_{\beta} V_{\beta}, \quad \theta(X, Y)=N_{\beta} \theta_{\beta}, \quad \theta_{s}(X, Y)=N_{\beta} \theta_{s_{\beta}}, \\
& P(X, Y)=H_{\lambda} P_{\lambda}, \text { where } \beta=1,2, \ldots \ldots, 6 \text { and } \lambda=1,2,3 .
\end{aligned}
$$

Substituting the element velocity components, the temperature and the pressure distributions in the Eqs. (2) - (5), the finite element equations can be written in the form

$$
\begin{aligned}
& K_{\alpha \beta \gamma}{ }_{x} U_{\beta} U_{\gamma}+K_{\alpha \beta \gamma} y V_{\gamma} U_{\gamma}+M_{\alpha \mu}{ }_{\alpha \mu} P_{\mu}+\frac{1}{R e}\left(S_{\alpha \beta^{x x}}+S_{\alpha \beta} y y\right) U_{\beta}=Q_{\alpha^{u}} \\
& K_{\alpha \beta \gamma}{ }^{x} U_{\beta} V_{\gamma}+K_{\alpha \beta y^{y}} V_{\gamma} V_{\gamma}+M_{\alpha \mu^{y}} P_{\mu}+\frac{1}{R e}\left(S_{\alpha \beta}{ }^{x x}+S_{\alpha \beta} y y\right) V_{\beta}-R i K_{\alpha \beta} \theta_{\beta}+\frac{H a^{2}}{R e} K_{\alpha \beta} V_{\beta}=Q_{\alpha^{v}} \\
& K_{\alpha \beta \gamma}{ }_{\alpha} U_{\beta} \theta_{\gamma}+K_{\alpha \beta \gamma}{ }^{y} V_{\beta} \theta_{\gamma}+\frac{1}{R e P r}\left(S_{\alpha \beta}{ }^{x X}+S_{\alpha \beta} y y\right) \theta_{\beta}-J K_{\alpha \beta} V_{\beta}^{2}=Q_{\alpha} \theta \\
& \frac{K}{\operatorname{Re} \operatorname{Pr}}\left(S_{\alpha \beta^{x X}}+S_{\alpha \beta} y y\right) \theta_{\beta}=Q_{\alpha} \theta_{S}
\end{aligned}
$$

The coefficients in element matrices are in the form of the integrals over the element area as

$$
\begin{aligned}
& K_{\alpha \beta^{x}}=\int_{A} N_{\alpha} N_{\beta, x} d A, \quad K_{\alpha \beta^{y}}=\int_{A} N_{\alpha} N_{\beta, y} d A, \quad K_{\alpha \beta \gamma^{x}}=\int_{A} N_{\alpha} N_{\beta} N_{\gamma, x} d A, \\
& K_{\alpha \beta \gamma} y=\int_{A} N_{\alpha} N_{\beta} N_{\gamma, y} d A, \quad K_{\alpha \beta}=\int_{A} N_{\alpha} N_{\beta} d A, \quad S_{\alpha \beta^{X X}}=\int_{A} N_{\alpha, x} N_{\beta, x} d A,
\end{aligned}
$$




$$
\begin{array}{lll}
S_{\alpha \beta}^{y y}=\int_{A} N_{\alpha, y} N_{\beta, y} d A, & M_{\alpha \mu^{x}}=\int_{A} H_{\alpha} H_{\mu, x} d A, & M_{\alpha \mu^{y}}=\int_{A} H_{\alpha} H_{\mu, y} d A, \\
Q_{\alpha}{ }^{u}=\int_{S_{0}} N_{\alpha} S_{x} d S_{0}, & Q_{\alpha}{ }^{\nu}=\int_{S_{0}} N_{\alpha} S_{y} d S_{0}, & Q_{\alpha} \theta=\int_{S_{w}} N_{\alpha} q_{1_{w}} d S_{w}, \\
Q_{\alpha} \theta_{s}=\int_{S_{w}} N_{\alpha} q_{2 w} d S_{w} . & &
\end{array}
$$

Using reduced integration technique of Reddy [12] and Newton-Raphson method of Roy and Basak [13], the set of non-linear algebraic Eqs. (6) - (9) are transferred into linear algebraic equations. Finally, these linear equations are solved by applying Triangular Factorization method of Zeinkiewicz et al. [14]. The application of this simulation is well described by Taylor and Hood [15] and Dechaumphai [16].

\subsection{Grid refinement check}

In order to decide suitable grid size for this analysis, a grid independence test is reported with $H a=12, R e=100, J=1, K=7, A=0.05, \lambda=2, D=0.2, R i=0.1$ and $\operatorname{Pr}=0.73$. The extreme values of the average Nusselt number $(\mathrm{Nu})$ that relates to the heat transfer rate of the heated surface and average temperature $\left(\theta_{a v}\right)$ of the fluid in the enclosure are used as sensitivity measures of the correctness of the solution. They are selected as the supervising variables for the grid liberty test. Table 1 expresses the addiction of the quantities $\mathrm{Nu}$ and $\theta_{a v}$ on the grid size and the computational time. By considering both the accuracy of numerical values and computational time, the current formulation is performed with 31514 nodes and 3636 elements grid system.

Table 1 . Grid sensitivity check at $H a=12, P r=0.73, R i=0.1, R e=100, A=0.05, K=7$, $\lambda=2, J=1$ and $D=0.2$.

\begin{tabular}{cccccc}
\hline $\begin{array}{c}\text { Nodes } \\
\text { (elements) }\end{array}$ & $\begin{array}{c}3918 \\
(436)\end{array}$ & $\begin{array}{c}9373 \\
(1064)\end{array}$ & $\begin{array}{c}15465 \\
(1768)\end{array}$ & $\begin{array}{c}31514 \\
(3636)\end{array}$ & $\begin{array}{c}49592 \\
(5748)\end{array}$ \\
\hline $\mathrm{Nu}$ & 1.59789 & 1.60277 & 1.61824 & 1.62264 & 1.62264 \\
$\theta_{a v}$ & 0.33624 & 0.32512 & 0.31054 & 0.30611 & 0.30611 \\
Time (s) & 246.269 & 308.603 & 398.167 & 445.321 & 550.379 \\
\hline
\end{tabular}

The model validation is a necessary part of a mathematical investigation. Hence, the outcome of the present numerical code is benchmarked against the numerical result of Rahman and Alim [17] which was reported for MHD mixed convection flow in a vertical lid-driven square enclosure including a heat conducting horizontal circular cylinder with Joule heating. The comparison is conducted while employing the dimensionless parameters $R e=100, R i=5, K=5, H a=10, J=1, D=0.2$ and $\operatorname{Pr}=0.71$. The present result for both the streamlines and isotherms is shown in Fig. 2, which shows an outstanding agreement 
with those of Rahman and Alim [17]. This justification boosts the assurance in this numerical code to carry on with the above stated objectives of the existing investigation.

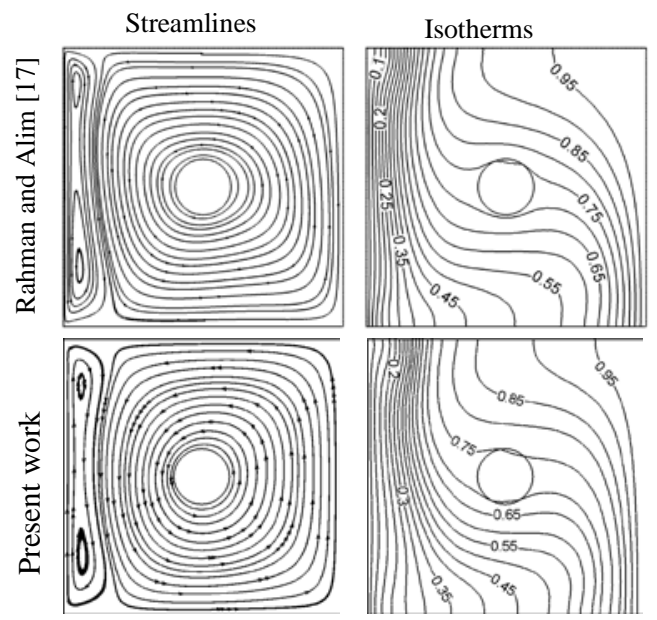

Fig. 2. Code validation for the present study.

\section{Results and Discussion}

The MHD combined convection phenomenon inside a lid driven square chamber having a heat conducting square cylinder is influenced by different controlling parameters such as $\mathrm{Ha}, \mathrm{K}, \mathrm{Re}, \mathrm{Pr}, \mathrm{J}$ and $\mathrm{Ri}$. Analysis of the results is made through obtained streamlines, isotherms, average Nusselt number, average fluid temperature and temperature at the cylinder centre for two parameters varied as $0 \leq J \leq 7$ and $0.1 \leq R i \leq 10$, while the other parameters $\mathrm{Re}, \mathrm{Ha}, \mathrm{K}$ and $\mathrm{Pr}$ are reserved fixed at 100,12, 7 and 0.73 , respectively.

Figs. 3 (a) - (b) depicts the flow and temperature fields in terms of computed streamlines and isotherms at the four representative values of the dimensionless Joule heating parameter $J(0,1,4$ and 7$)$ and in the absence of free convection effect $(R i=0.1)$. The flow exhibits a simple clockwise recirculation pattern, which becomes concentrated along the cold moving wall for all values of $J$. On the other hand, conductive distortion of isothermal lines starts to appear at the vicinity of the heated surface and upper part of the cavity at the lower values of $J(0$ and 1$)$. Thinner boundary layer is developed to form near the cold wall with a clear indication of the existence of thermal spots with rising $J$. The contour lines mimic the wall's profile in the neighbourhood of the vertical wavy surface.

Increasing the convective parameter $R i$ to 1 , corresponding streamlines and isotherms are exposed in Figs. 4 (a) - (b). The velocity field contains two counter rotating eddies in the cavity for all $J$. That means the fluid flow is established by a relatively balanced interaction of the two driving mechanisms. The isothermal lines at the two lower values of 
$J$ are nearly parallel to the vertical walls of the cavity, which gives a clear indication of conduction and combined convection dominated heat transfer in the chamber. For higher $J$ (4 and 7), they start to turn back (convective distortion) towards the left lid near the top wall due to the dominating influence of the convective current. The thermal boundary layer becomes less compacted and isothermal lines occupy the bulk of the chamber due to the variation of $J$.

(b)
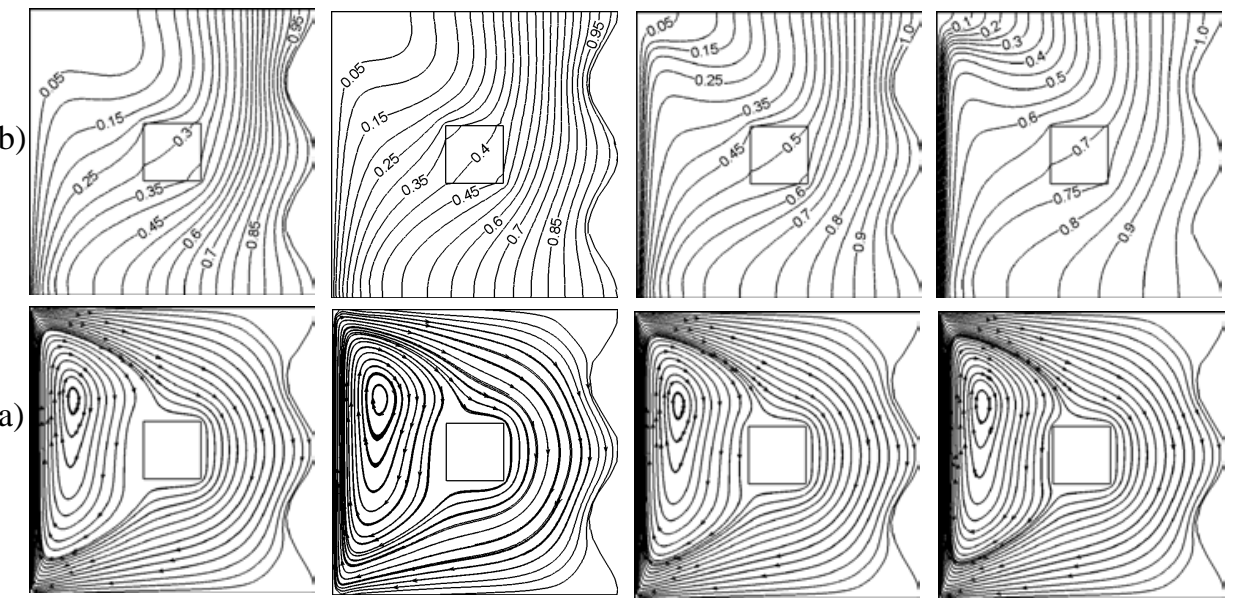

$J=1$

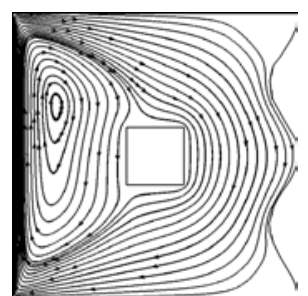

$J=4$

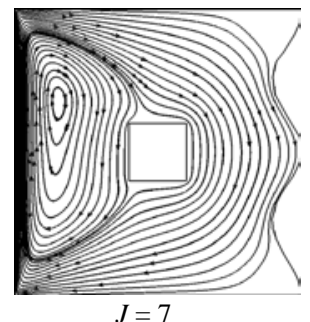

$J=7$

Fig. 3. Effect of $J$ with $R i=0.1$ on (a) Streamlines and (b) Isotherms.

(b)
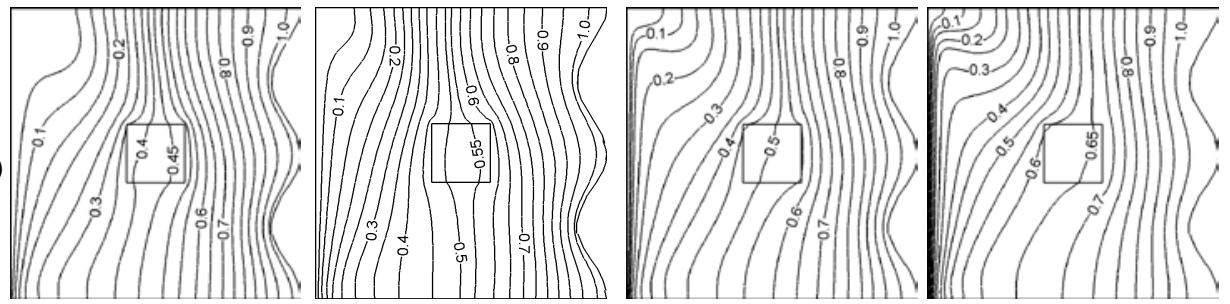

(a)

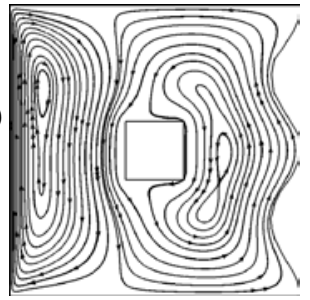

$J=0$
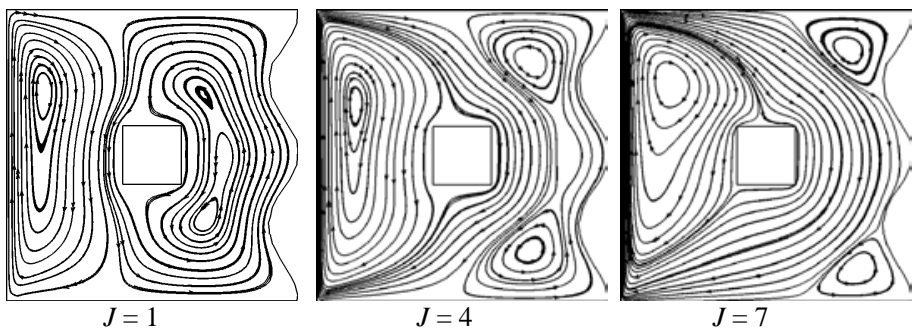

Fig. 4. Effect of $J$ with $R i=1$ on (a) Streamlines and (b) Isotherms. 
On further increasing $R i$ up to 10 (purely free convection regime) as illustrated in Figs. 5 (a)-(b), the counter clockwise circulating cell on the right grows further and occupies most of the part of velocity field at $J=0$. As a result the clockwise cell becomes weaker and smaller. A similar phenomena is observed for $J=1$. However, at $J=4$, another small eddy is developed at the right top corner in the cavity. Further elevation in $J$ creates two small vortices near the wavy surface in the velocity field. The convective distortion of isothermal lines occurs throughout the cavity for all $J$ due to the strong influence of the convective current. Consequently, the isotherms depart more from the hot sinusoidal corrugated wall and begin to crowd near lid forming a thick thermal boundary layer as compared to the previous case.

(b)
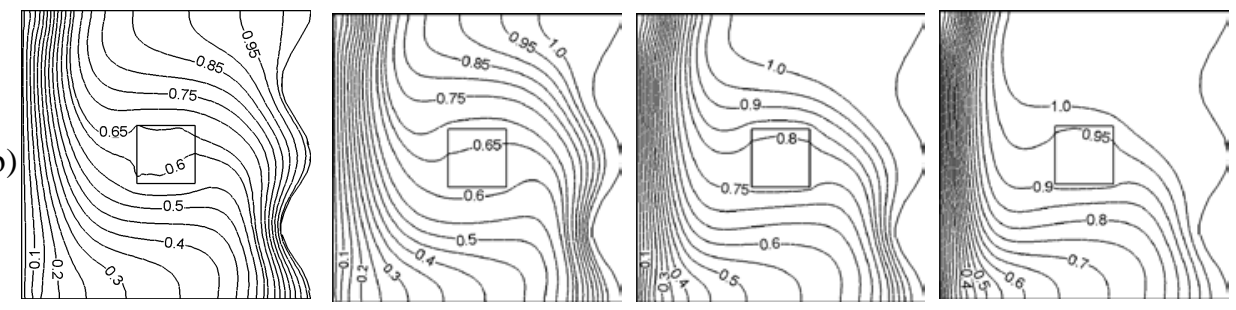

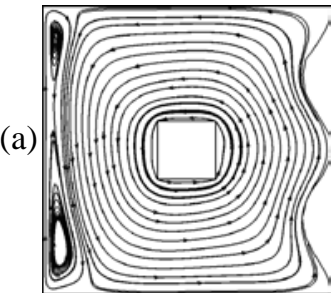

$J=0$

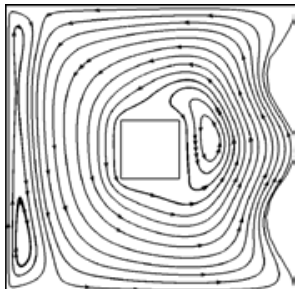

$J=1$

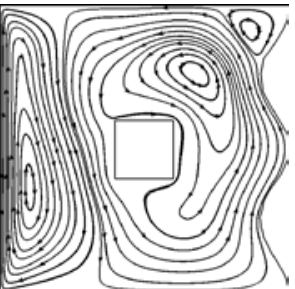

$J=4$

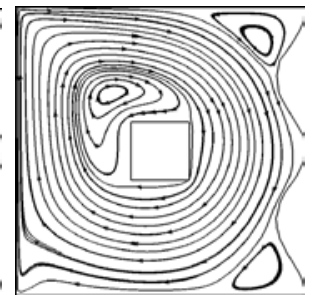

$J=7$

Fig. 5. Effect of $J$ with $R i=10$ on (a) Streamlines and (b) Isotherms.

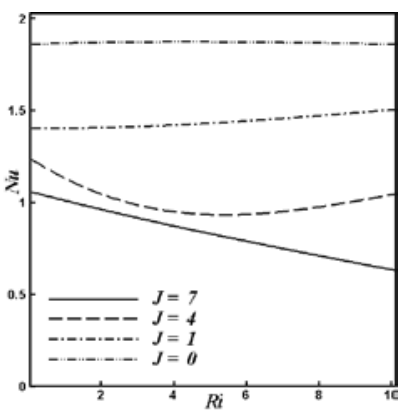

(i)

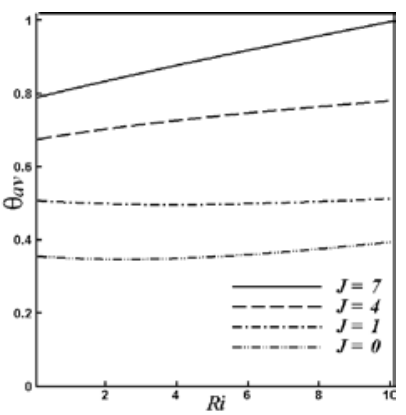

(ii)

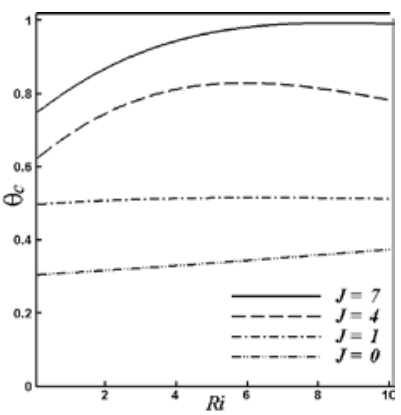

(iii)

Fig. 6: Effect of $J$ on (i) $N u$, (ii) $\theta_{a v}$ and (iii) $\theta_{c}$ for various $R i$. 
In order to evaluate how the presence of Joule heating affects the heat transfer rate along the hot wall, average Nusselt number $(\mathrm{Nu})$ is plotted as a function of $\mathrm{Ri}$ in Fig. 6. $\mathrm{Nu}$ becomes independent of Ri for $J=0$ and grows very slowly at $J=1$. When $J=4, N u$ devalues up to $R i=4$, and then increases gradually with $R i$. Further, it is reduced with escalating $R i$ for the highest value of $J$. It is also noted that $N u$ is always higher at $J=0$, which is expected. In addition, Fig. 6 explains the average temperature of the fluid $\left(\theta_{a v}\right)$ in the chamber and temperature at the cylinder center $\left(\theta_{c}\right)$ as a function of $R i$. At the upper two values of $J$ ( 4 and 7), they grow up rapidly and for the remaining $J$, they become almost constant with rising $R i$.

\section{Conclusion}

A computational study is performed to consider the MHD combined convection flow in a lid driven chamber with a heat conducting horizontal square cylinder. Results are obtained for wide ranges of Joule heating parameter and Richardson number. The following conclusions may be drawn from the current analysis:

- The variation in $J$ does not affect significantly the streamlines in the pure forced convection region, but has noteworthy effect in the combined and free convection dominated regimes. However the thermal current activities have been changed dramatically.

- The raise in $R i$ exposes different flow patterns creating more secondary eddies near the sinusoidal surface and a thick thermal boundary layer is developed near the lid driven surface.

- $\quad \theta_{a v}$ and $\theta_{c}$ are obtained maximum for the highest value of $J$ with mounting Ri. But it negates the heat transfer from the heated surface.

\section{References}

1. T. H. Hsu and S. P. How, Acta Mechanica 133, 87 (1999). http://dx.doi.org/10.1007/BF01179012

2. A. Omri, and S.B. Nasrallah, Numer. Heat Trans. (Part A) 36, 615 (1999). http://dx.doi.org/10.1080/104077899274606

3. A. J. Chamkha, Numer. Heat Transfer (Part A) 41, 529 (2002). http://dx.doi.org/10.1080/104077802753570356

4. C. -C.Wang, and Chen, C.-K. Int. J. Heat Mass Trans. 45, 2587 (2002). http://dx.doi.org/10.1016/S0017-9310(01)00335-0

5. G. Gau and M.A.R. Sharif, Int. J. Therm. Sci. 43 (5), 465 (2004). http://dx.doi.org/10.1016/j.ijthermalsci.2003.08.008

6. H. Shokouhmand and H. Sayehvand, Int. J. Eng. (Transactions A): Basics 17, 301 (2004).

7. M. T. Bhoite, G. S. V. L Narasimham, and M. V. K Murthy, Int. J. Therm. Sci. 44, 121 (2005). http://dx.doi.org/10.1016/j.ijthermalsci.2004.07.003

8. A. Al-Amiri, K. Khanafer, J. Bull, Ioan Pop, Int. J. Heat Mass Trans. 50, 1771 (2007). http://dx.doi.org/10.1016/j.jiheatmasstransfer.2006.10.008

9. R. Nasrin, Int. Commun. Heat Mass Trans. 38, 1269 (2011). http://dx.doi.org/10.1016/j.icheatmasstransfer.2011.06.005

10. R. Nasrin, J. Sci. Res. 3 (3), 501 (2011). http://dx.doi.org/10.3329/jsr.v3i3.7433 
11. M. M. Rahman, M. A. Alim, M. M. A. Sarker, Int. Commun. Heat Mass Trans. 37, 524 (2010). http://dx.doi.org/10.1016/j.icheatmasstransfer.2009.12.012

12. J. N. Reddy, An Introduction to Finite Element Analysis (McGraw-Hill, New York, 1993).

13. S. Roy and T. Basak, Int. J. Eng. Sci. 43 (8 - 9), 668 (2005).

14. O.C. Zeinkiewicz, R. L. Taylor, J. M. Too, Int. J. Num. Meth. Eng. 3, 275 (1971). http://dx.doi.org/10.1002/nme.1620030211

15. C. Taylor and P. Hood, Computers and Fluids, 1 (1), 73 (1973). http://dx.doi.org/10.1016/00457930(73)90027-3

16. P. Dechaumphai, Finite Element Method in Engineering, 2nd ed. (Chulalongkorn University Press, Bangkok, 1999).

17. M. M. Rahman and M. A. Alim, Nonlinear Analysis: Modelling and Control 15 (2), 199 (2010). 